\title{
Guide Device
}

National Cancer Institute

\section{Source}

National Cancer Institute. Guide Device. NCI Thesaurus. Code C49974.

A device designed to help direct the passage of another object. 